\title{
Genetic Characterisation and Cytological Identification of a Male Sterile Mutant in Maize (Zea mays L.)
}

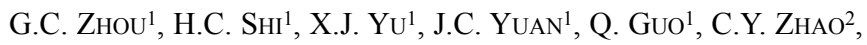 \\ Q. $\mathrm{SUN}^{3}$ and Y.P. $\mathrm{KE}^{1,2^{*}}$ \\ ${ }^{1}$ College of Agronomy, Sichuan Agricultural University, Chengdu, Sichuan 611130, China \\ ${ }^{2}$ Sichuan Nongda Zhenghong Bio. Co., Ltd, Chengdu, Sichuan 610213, China \\ ${ }^{3}$ College of Life Sciences, Key laboratory of Bio-resources and Eco-environment \\ of the Ministry of Education, Sichuan University, Chengdu, Sichuan 610064, China
}

(Received 18 March 2017; Accepted 24 August 2017;

Communicated by M. Molnár-Láng)

\begin{abstract}
Male sterile mutants play an important role in the utilisation of crop heterosis. Male sterile plants were found in $\mathrm{S}_{5}$ generations of maize hybrid $\mathrm{ZH} 2$, through continuous sib-mating by using the fertile plants in the same population, we obtained a male sterile sibling population K932MS including sterile plants K932S and a fertile plant K932F. The objective of this study was to clarify the genetic characterisation and abortion characteristics by nucleus and cytoplasm effect analyses, cytoplasm grouping, and cytological observation. The results showed that no difference was found between K932S and K932F in the vegetative growth stage, but K932S had no emerging anther or pollen grains. The segregation ratio of fertile plants to sterile plants was $1: 1$ in the sibling progenies, while it was 3:1 in self-crossing progenies of $\mathrm{K} 932 \mathrm{~F}$. The sterility of $\mathrm{K} 932 \mathrm{~S}$ could be restored among reciprocal progenies when seven normal inbred lines were used as females respectively. The fertility expression of K932S crossed with 30 testers would be changed in different test-crosses and some backcross progenies. The C-type restorer Zifeng-1 (Rf4Rf4) was able to restore the fertility of K932S, and the specific PCR amplification bands of K932MS were consistent with CMS$\mathrm{C}_{\mathrm{Mo17}}$. The anther of K932S began abortion at dyad with its tapetum expanded radically and vacuolated: this induced abnormality in the shapes of both dyads and tetrads. The microspore could not develop normally, and then it collapsed and gradually disappeared. Hence, K932MS is a C-type cytoplasmic male sterile mutant with a pollen-free, stable inheritance: it has potential application value for further research.
\end{abstract}

Keywords: maize, male sterile, genetic analysis, cytoplasmic identification, tapetum

\section{Introduction}

In the production of hybrid seeds of maize (Zea mays L.), male sterility (MS) properties are beneficial to preventing artificial emasculation, reducing production costs of seeds, and improving seed purity, which provide an important basis for the effective utilization of heterosis in maize (Duvick 1958). Also, male sterile materials are widely used to study

\footnotetext{
*Corresponding author. E-mail: keyp169@163.com. Tel: +862867085333
} 
substance metabolism and molecular mechanisms (Nan et al. 2017) of anther development.

In general, MS can be classified into two types: genetic male sterility (GMS) and cytoplastic male sterility (CMS). In the earliest reports, the mutant of GMS in maize was named $m s 1$ (Eyster 1921). Whereafter, more than 40 recessive GMS genes controlled by single genes have been disclosed and mapped (Maize GDB, www.maizegdb.org), some of which were later cloned (Moon et al. 2013). Although, $m s 45$ has been applied to the production and breeding of maize via seed production technology (SPT) (Wu et al. 2015), there are segregated sibling populations of GMS, making it difficult to obtain a stable sterile line. Accordingly this deficiency restricts the usefulness of more genetic male sterile resource.

In fact, CMS materials were first used for the hybrid production of maize. CMS is divided into C, S and T types. Rhoades (1933) firstly found a T-type CMS material of maize and applied it in the 1950s, with the efficiency of hybrid seed production and maize yield improving greatly. However, because of the specialised infection of Helminthosporium maydis race T (Levings 1990), maize production in the United States suffered significant damage in 1970. Moreover, different types of CMS call for distinct restorer genes for their fertility. For example, the fertility restoration of CMS-T is controlled by two dominant common genes of $R f 1$ and $R f 2$ (Cui et al. 1996), while that of CMS-S is controlled by $R f 3$ and $R f^{*}$ (Kamps and Chase 1997), respectively. Moreover, the fertility restoration of CMS-C is determined by its major gene $R f 4$ (Sisco 1991). Recently, another new major restorer gene $R f^{*}-A 619$ was reported (Liu et al. 2016). Although $R f 5$ can restore the fertility of CMS-C, RfI inhibits this restoration (Hu et al. 2006), $R f 6$ shows a weak ability in fertility restoration, which is easily affected by the environment (Qin et al. 1990). Beckett (1971) proposed an identification method according to the special effects of fertility restoration to distinguish the types of CMS. With the development of molecular technology, the mitochondrial DNAs (mtDNAs) of the three types of CMS have different specificities (Allen et al. 2007). By using specific primers, cytoplasmic types of CMS of maize can be identified rapidly and accurately by using the PCR method (Liu et al. 2002).

Although breeders have obtained a variety of GMS and CMS materials from maize through various means, very few of them are used in practical applications: the CMS materials having single cytoplasmic genetic bases, and their being likely to have specialised infections such as Helminthosporium maydis race T or unstable MS (Warmke and Lee 1978), means that their application potential is limited. Therefore, it is of great significance to breed more new male sterile materials of maize from different sources with complete, stable, abortion potential. In this study, the genetic analysis, cytological observation, special restoration grouping identification, and specific cytoplasmic PCR identification were conducted on a male sterile mutant obtained recently, with the aim of identifying the genetic pattern and characteristics of the abortive nature of this material and provide a theoretical basis for further research and utilisation. 


\section{Materials and Methods}

\section{Plant materials}

We collected three different elite maize hybrids planted extensively at Southwestern China in spring, 2007. The three hybrids are high yield, fine quality and high resistance to multi- diseases, named them as $\mathrm{ZH} 1, \mathrm{ZH} 2$ and $\mathrm{ZH} 3$ respectively. We used them as the base populations to breed inbred lines by pedigree method. We firstly planted them as $\mathrm{S}_{0}$ at Lingshui $\left(109^{\circ} 30^{\prime} \mathrm{E}, 18^{\circ} 15^{\prime} \mathrm{N}\right)$, Hainan Province, China, in autumn, 2007. Then the elite plants were chosen to self-pollination and planted $\mathrm{S}_{1}$ populations at Shuangliu $\left(103^{\circ} 56^{\prime} \mathrm{E}, 30^{\circ} 34^{\prime} \mathrm{N}\right)$, Sichuan Province, China, in spring, 2008. Repetitive the work until we found six spontaneous mutation male sterile plants in the $\mathrm{S}_{5}$ self-pollination population derived from $\mathrm{ZH} 2$, and utilised fertile pollen crossed with sterile plants in the same population to construct a sibling population, at Shuangliu, in spring, 2010. We selected the segregation sibling population before proceeding with sib-mating, for which the sibling population was planted at Lingshui, in autumn, 2010. Then, fertile plants that had been sib-mated several times with male sterile plants at the two aforementioned locations from 2011 to 2012, were taken as the sibling population (named K932MS), with the sterile plants named K932S and the fertile plants named K932F, therein.

We planted combination $\mathrm{F}_{1}$ which was prepared from K932S and 30 testers (Table S1*), K932S $\times$ K932F, and the self-progeny of K932F, in Sichuan Province, China, in spring, 2013. In addition, the sterile plants in seven $F_{1}$ combinations were selected as female parents and the corresponding seven testers were used as male parents to backcross seven $\mathrm{BC}_{1}$ populations; furthermore, by using seven testers as female parents, a complete fertile plant in the $\mathrm{F}_{1}$ combination was used as the male parent to construct $\mathrm{F}_{1}$ reciprocal cross-populations at Hainan Province, China, in autumn, 2013. The sterile plants in each $\mathrm{BC}_{1}$ were used as female parents to backcross continuously into $\mathrm{BC}_{2}$ populations, and construct $\mathrm{F}_{2}$ reciprocal cross-populations at Sichuan Province, China, in spring, 2014. The aforementioned materials were provided by Sichuan Nongda Zhenghong Bio. Co. Ltd., China. The sterile materials: CMS- $\mathrm{T}_{\mathrm{Mo17}}, \mathrm{CMS}-\mathrm{C}_{\mathrm{Mo17}}$, and $\mathrm{CMS}-\mathrm{S}_{\mathrm{Mo17}}$ and the maintainer line Mo17, test lines Hui313 (Rf3Rf3) and Zifeng-1 (Rf4Rf4) for the test systems were obtained from The Maize Research Institute of Sichuan Agricultural University, Chengdu, Sichuan Province, China.

\section{Fertility identification}

By identifying the fertility in the field based on Duvick's five-grade classification standard (Duvick 1965). From the beginning to the end of pollen dispersal, the fertility of the plants was investigated every day. The mature anthers were dyed for five minutes using $1 \% \mathrm{I}_{2}$-KI and then the dye-ability of the pollen was tested under a microscope and recorded.

\footnotetext{
*Further details about the Electronic Supplementary Material (ESM) can be found at the end of the article.
} 


\section{Analysis of nuclear effects}

From 2013 to 2014, K932S $\times$ K932F and the self-progeny of K932F were planted to investigate fertility segregation in different places, including Lingshui in Hainan Province, China; Shuangliu in Sichuan Province, China; and Tieling (123 $\left.83^{\prime} \mathrm{E}, 42^{\circ} 28^{\prime} \mathrm{N}\right)$ in Liaoning Province, China. A $\chi^{2}$ test was conducted to test the fertility segregation ratios of the populations. In addition, the nuclear effects and fertility stability were analysed.

\section{Analysis of cytoplasmic effects}

From 2014 to $2015, F_{1}$ and $F_{2}$ reciprocal populations were planted to investigate fertility characteristics and analyse any cytoplasmic effects, in both Shuangliu in Sichuan Province and Lingshui in Hainan Province.

\section{Analysis of nucleo-cytoplasmic interaction effects}

From 2013 to 2015 , the $30 \mathrm{~F}_{1}$ populations above and seven $\mathrm{BC}_{1}, \mathrm{BC}_{2}$ populations were planted to observe the fertility performances and analyse nucleo-cytoplasmic interaction effects therein.

\section{Identification methods based on special restoration effects}

According to the identification method proposed by Zheng (1982), K932S was test crossed with Hui313 (Rf3Rf3) and Zifeng-1 (Rf4Rf4) for restoring the test lines to observe the fertility performances of the test cross progenies.

\section{Grouping identification based on PCR amplification with special primers}

In the period in which the plants had three leaves and one half leaf, the total DNAs of K932S, K932F, CMS-T ${ }_{\text {Mo17 }}$, CMS-C ${ }_{\text {Mo17 }}$, and $\mathrm{CMS}_{-S_{M o 17}}$, and maintainer line Mo17 were extracted for PCR amplification with special primers. The special primers and the PCR conditions referred to Slischuk et al (2011). Thereafter, electrophoresis detection was carried out for the PCR products on a $2 \%$ agarose gel.

\section{Paraffin section of anthers}

Each plant in the K932MS was labelled. From the development of male flowers to complete anthesis, some florets on the cob of the male tassel of each plant in the sister crosspopulation were collected every other day to be fixed in FAA stationary liquid and then reserved in $70 \%$ alcohol at $4{ }^{\circ} \mathrm{C}$. Other florets were retained on each plant for identifying fertility when pollens were dispersed. The florets were sliced (using a KD-202A slicing machine) through regular paraffin sectioning method to a thickness of $8 \mu \mathrm{m}$. After being 
dyed using hematoxylin-eosin, the slices were sealed by neutral balsam and then observed and photographed under a Nikon 80i microscope.

\section{Anther tablet}

By using a compression method, the preserved anthers were dyed with $0.5 \%$ carmine acetate to observe the development of microspores under the Nikon 80i microscope.

\section{Results}

\section{Male tassel characteristics of the sterile mutant}

By comparing the male tassels of fertile and sterile plants in the sibling population, it is found that the K932S and K932F male tassels were of a similar shape: with obvious main tassels and few lateral branches (Fig. S1a). The yellowish green, plump anther in the male tassels of fertile plant K932F was exposed and dispersed pollens normally; however, the florets of sterile plant K932S did not crack and the anther was wizened (Fig. S1b and Fig. $\mathrm{S} 1 \mathrm{c})$. By using $\mathrm{I}_{2}-\mathrm{KI}$ dying, the pollen grains in the K932F anther were dyed dark blue, while pollen grains were not found in the K932S anther (Fig. S1d). This indicated that K932S exhibited pollen-free male sterility, and abortive characteristics.

\section{Analysis of nuclear effects}

To identify the genetic characteristics of $K 932 M S$. K932MS, and the self-progeny of K932F (Table S2), they were planted in different ecological environments from 2013 to 2014. K932MS was deemed sterile in different ecological environments, indicating that the sterility of K932MS was stably genetic and unaffected by natural conditions. The segregation ratio of fertile plants to sterile plants was $1: 1$ in $K 932 M S$, while it was 3:1 among self-progeny of K932F, being, as it was, subjected to genetic modes of single genes. It is shown that the sterile characteristics were controlled by a pair of homozygous recessive genes, while fertility was controlled by heterozygous and dominant homozygous genes.

\section{Analysis of cytoplasmic effects}

Selecting seven testers with normal cytoplasm as female parents and the completely fertile plants of $\mathrm{K} 932 \mathrm{~S} \times \mathrm{A} 9-2$ as male parents, the $\mathrm{F}_{1}$ reciprocal cross-population was prepared and the results showed that all $\mathrm{F}_{1}$ reciprocal cross-population members were fertile. Then the $\mathrm{F}_{2}$ reciprocal cross-populations were also all fertile, determining that the sterile characteristics of $K 932 \mathrm{MS}$ were affected by maternal inheritance and that their cytoplasm contained sterile genes. 


\section{Analysis of cytoplasm-nuclear interaction effects}

To identify whether, or not, K932MS sterility was affected by cytoplasm-nuclear interaction effects, K932S was taken as a female parent crossed with 30 testers (Table S1), and the $\mathrm{F}_{1}$ combinations were planted in three different locations. The fertility results demonstrated that the fertility performances of $F_{1}$ combinations were consistent in different places (Table S3). Tester 41 was able to restore the fertility of K932S and retain a stable fertility, while the other 21 testers, including A9-5, were able to keep K932S sterile. Furthermore, the other eight testers partially, or in an unstable manner, restored the fertility of K932S. Different male testers, when crossed to K932S, can result in hybrids with different fertility characteristics, as the restorer genes for CMS are coded in the nucleus, indicating that the fertility was affected by nucleo-cytoplasmic interactions.

By selecting sterile plants in the seven $\mathrm{F}_{1}$ combinations as female parents and the corresponding testers as male parents, the first backcross progeny $\mathrm{BC}_{1}$ was obtained through backcrossing. To identify $\mathrm{BC}_{1}$ fertility, it was found that the $\mathrm{BC}_{1}$ population of testers QIII-1 and SYD1-1 with K932S showed different fertility restoration abilities. The other five $\mathrm{BC}_{1}$ populations were found to be thoroughly abortive (Table S4). Compared with Table S3, when the nuclear background of testers QIII-1, SYD1-1, and A9-2 in the testcrossed progenies was changed, the fertility of their progenies gave contrasting performances. This indicated that the fertility of $K 932 M S$ was influenced by different nuclear backgrounds and when the nuclear genes were not homozygous, the fertility segregation appeared due to the effects of nucleo-cytoplasmic interaction.

By using the sterile plants in $\mathrm{BC}_{1}$ as female parents, backcrossing was carried out once again to obtain $\mathrm{BC}_{2}$. The fertility performances of $\mathrm{BC}_{2}$ are listed in Table $\mathrm{S} 5$. Compared with the fertility of $\mathrm{BC}_{1}$, the $\mathrm{BC}_{2}$ population obtained from QIII-1 and SYD1-1 underwent complete abortion and other $\mathrm{BC}_{2}$ populations were sterile, or nearly so. This further demonstrated that the change in nuclear background would alter the fertility: there are nucleo-cytoplasmic interaction effects in $K 932 M S$ and in conclusion, we can explicitly state that $\mathrm{K} 932 \mathrm{MS}$ is nucleo-cytoplasmic male sterile.

\section{Grouping identification based on special restoration effects analysis}

The testcross $\mathrm{F}_{1}$ from tester Hui313 (Rf3Rf3) crossed with K932S was completely sterile, showed that K932MS is not S-type CMS; while testcrossed $\mathrm{F}_{1}$ of Zifeng-1 (Rf4Rf4) and $\mathrm{K} 932 \mathrm{~S}$ was fertile, indicating that K932MS could be categorised as a C-type CMS.

\section{Identification through PCR amplification with specific primers analysis}

PCR amplification was performed with specific primers on the total DNAs of K932S, $\mathrm{K} 932 \mathrm{~F}$ and CMS- $\mathrm{C}_{\mathrm{Mo17}}, \mathrm{CMS}-\mathrm{S}_{\mathrm{Mo17}}, \mathrm{CMS} \mathrm{T}_{\mathrm{Mo17}}$, and maintainer line Mo17. The results (Fig. S2) show that, when PCR with C-type specific primer, CMS-C $\mathrm{Mo17}_{\text {, }}$ K932S, and K932F had the same amplification bands, their size was $398 \mathrm{bp}$; however, when the Sand T-types of specific primers were used for PCR, the corresponding bands were only 
found in CMS-S $\mathrm{S}_{\mathrm{Mo17}}$ and CMS- $\mathrm{T}_{\mathrm{Mo17}}$. Therefore, this further demonstrated that K932MS was of the CMS-C type.

\section{Observation of transverse section of anthers}

Through observation of transverse section of K932F and K932S anthers by using a paraffin sectioning method, the results showed that during the pollen mother cell period, the anther sac was composed of four layers of cells and pollen mother cells, and fertile and sterile anthers had identical structures (Fig. S3a and Fig. S3e). Then, the pollen mother cells changed to become elliptical (Fig. S3b and Fig. S3f), followed by the first meiosis and the formation of dyads (Fig. S3c and Fig. S3g). The tapetum in the fertile anther was compressed (Fig. S3c), while that in the sterile anther expanded radially and was vacuoles (Fig. S3g). When the tetrad was separated, the fertile microspores developed into pollen grains normally (Fig. S3d), and the tapetum of the sterile anther expanded continuously and filled the whole anther cell and then any microspores disappeared (Fig. S3h).

\section{Observation of microspore development}

The development of microspores in the anther of plants K932F and K932S was observed using a compression method. The results demonstrated that the shapes of fertile and sterile pollen mother cells were similar, indicating the normality of sterile pollen mother cells (Fig. S4a and Fig. S4e). Furthermore, the sterile pollen mother cells were in meiosis and formed dyads and tetrads with irregular shapes (Fig. S4f and Fig. S4g). In particular, the tetrads were disintegrated (Fig. S4g) and formed microspores with irregular shapes which were smaller than normal microspores (Fig. S4h). Finally, the tetrads developed into mature pollen grains. Therefore, the abortion of $K 932 M S$ was considered to begin from the dyad and last to the autolysis and disintegration of young microspores.

\section{Discussion}

\section{Genetic characterisation of the K932MS}

The MS phenomenon of plants commonly seen in flowering plants (Kaul 1988) is controlled by genes. However, some reports pointed out that the MS phenomenon is regulated by the environment, such as in NK58S (Ding et al. 2012) and PA64S (Zhou et al. 2012) in rice, and temperature-sensitive Qiong68ms (Tang et al. 2006) in maize. This research found that K932MS had stable MS in different growing seasons and ecological environments. This indicates that the sterility of K932MS is stably genetic and unaffected by changes in temperature, light, and latitude. A majority of sterile characteristics of nuclear of maize are controlled by recessive single genes, while only a few including $M s 41$, Ms42, Ms44, etc. are controlled by dominant genes (Skibbe and Schnable 2005). In this study, the proportion of fertile and sterile plants in $K 932 M S$ was $1: 1$, while that of the self-progeny of $\mathrm{K} 932 \mathrm{~F}$ was $3: 1$, indicating that the sterility of $K 932 \mathrm{MS}$ was controlled by 
a pair of nuclear recessive genes. The reciprocal cross progenies $F_{1}$ and $F_{2}$, utilising testers with normal cytoplasm as female parents, were all fertile, showing that the sterility of $K 932 M S$ was affected by cytoplasmic inheritance. By using the testers in normal cytoplasm to test cross with sterile plants, it was found that the progenies showed complete fertility restoration, complete sterility, and partial fertility restoration, when different male testers were crossed to K932S, the fertility of K932S would also be altered, which indicated that the fertility of $K 923 \mathrm{MS}$ was influenced by nuclear-cytoplasm interaction. Hence, it could be determined that K932MS is a nucleo-cytoplasmic interaction male sterile mutant.

The nucleo-cytoplasmic interaction male sterility of maize included $\mathrm{C}, \mathrm{S}$, and $\mathrm{T}$ types. Each type had its own special restoration genes, such as, $R f 2, R f 3$, and $R f 4$ which were able to restore the fertility of the CMS-T type (Dewey and Levings 1987), CMS-S type (Zabala et al. 1997), and CMS-C type (Ren et al. 2012), respectively. This study found that Hui313 (Rf3Rf3) maintained the sterility of K932MS, while Zifeng-1 (Rf4Rf4) restored the fertility of $K 932 \mathrm{MS}$, inferring that $K 932 \mathrm{MS}$ belonged to C-type CMS. Research in recent years shows that mtDNA is the genetic carrier (Hu et al. 2014) for the occurrence of CMS, and that various plants in different types of cytoplasm contained mtDNA with distinct structures and sequences (Allen et al. 2007; Alverson et al. 2011; Chen and Liu 2014). At present, specific primers for identifying cytoplasmic types have been found in plants including rice (Luan et al. 2013), maize (Slischuk et al. 2011), rape (Zhao et al. 2010), etc. By using mtDNA specific primers for PCR identification, this study found that

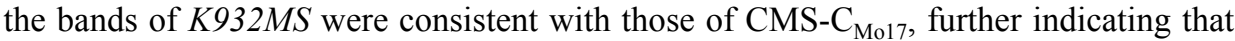
K932MS is a C-type cytoplasmic sterile mutant. The difficulty of application of C-type cytoplasmic sterile mutants lies in obtaining the necessary restorer: we have transferred its sterility trait to the existing excellent maize inbred lines, and screened its strong restorer lines, therefore showing that K932MS has application value for further research.

\section{Abortion characteristics of the K932MS mutant}

Although male flower development in plants is a complex process, the development of normal male flowers is roughly the same throughout (Wilson and Zhang 2009). Previous studies show that the pollens of C-, T-, and S-type CMS materials of maize exhibited different abortion characteristics (Colhoun and Steer 1981). The abortion period of CMS-S type of pollens was later, indicating that, while developing to bi-nucleate pollens, microspores suddenly collapsed, autolyzed, and disintegrated, but the cells in each layer remained normal.

Due to different nuclear backgrounds, the abortion of CMS-T type materials showed two phenomena: during meiosis, the pollen mother cells could not differentiate normally and therefore disintegrated; in the late monokaryotic stage, the tapetum became thickened and vacuolated, which postponed the disintegration, resulting in the abortion of pollens (Wise et al. 1999). Two types of abortion existed in CMS-C types as well: firstly, as tetrads were bonded into blocks and were thus inseparable, abortion occurred; secondly, owing to the irregular shape of the dyads the tapetum extended radially and vacuolated. 
In the stage of monokaryotic microspores, most microspores disintegrated and autolysed, thus complete abortion occurred (Lee et al. 1979). In the early stage of dyads, cells in each layer of K932MS anthers were normal, after the formation of dyads, however, the tapetum expanded radially and was vacuoles to the centre of the anther cells. Afterwards, the shapes of those tetrads thus were formed abnormal and the released microspores gradually disintegrated and autolysed.

Over the whole process, tapetum continuously expanded and filled the whole anther cell. This did not completely coincide with the abortion of CMS-C type materials found by Lee et al (1979), who discovered that vacuolisation of the tapetum appeared in the early stage of tetrad formation, while the vacuolisation of the tapetum of K932S occurred in the stage of dyad formation, probably due to the different nuclear backgrounds. Tapetum, which provides nutrients for the development of microspores, is critical to the development of pollens (Ma 2005): either advanced, or delayed, and tapetum disintegration can cause abortion of microspores. This has been reported in plants including Arabidopsis (Gu et al. 2014) and rice ( $\mathrm{Li}$ et al. 2015). This study found that the tapetum of K932S was vacuolated and disintegrated at a relatively later time, which was the main reason for the abortion of microspores.

\section{Acknowledgements}

This research was supported by the Maize Breeding Key Project (2011-3-11), the Science and Technology Support Programme (2016NYZ0006) and The Applied Fundamental Research Fund (2016JY0016) of Sichuan Province, China.

\section{References}

Allen, J.O., Fauron, C.M., Minx, P., Roark, L., Oddiraju, S., Lin, G.N., Meyer, L., Sun, H., Kim, K., Wang, C.Y., Du, F.Y., Xu, D., Gibson, M., Cifrese, J., Clifton, S.W., Newton, K.J. 2007. Comparisons among two fertile and three male-sterile mitochondrial genomes of maize. Genetics, 177:1173-1192.

Alverson, A.J., Zhuo, S., Rice, D.W., Sloan, D.B., Palmer, J.D. 2011. The mitochondrial genome of the legume vigna radiata and the analysis of recombination across short mitochondrial repeats. Plos One, 6:e16404.

Beckett, J.B. 1971. Classification of male sterile cytoplasms in maize (Zea mays L). Crop Sci. 11:724-727.

Chen, L.T., Liu, Y.G. 2014. Male sterility and fertility restoration in crops. Annu. Rev. of Plant Biol. 65:579606.

Colhoun, C.W., Steer, M.W. 1981. Microsporogenesis and the mechanism of cytoplasmic male sterility in maize. Ann. Bot. 48:417-424.

Cui, X.Q., Wise, R.P., Schnable, P.S. 1996. The $r f 2$ nuclear restorer gene of male-sterile T-cytoplasm maize. Science. 272:1334-1336.

Dewey, R.E., Levings, C.S. 1987. A mitochondrial protein associated with cytoplasmic male sterility in the T cytoplasm of maize. Proc. Nat. Acad. Sci. USA. 84:5374-5378.

Ding, J.H., Lu, Q., Ouyang, Y.D., Mao, H.L., Zhang, P.B., Yao, J.L., Xu, C.G., Li. X.H., Xiao, J.H., Zhang. Q.F. 2012. A long noncoding RNA regulates photoperiod-sensitive male sterility, an essential component of hybrid rice. Proc. Nat. Acad. Sci. USA 109:2654-2659.

Duvick, D.N. 1958. Yields and other agronomic characteristics of cytoplasmically pollen sterile corn hybrids, compared to their normal counterparts1. Agron. J. 50:121-125.

Duvick, D.N. 1965. Cytoplasmic pollen sterility in corn. Adv. in Genet. 13:1-56.

Eyster, L.A. 1921. Heritable characters of maize: VII. male sterile. J. Hered. 12:138-141. 
Gu, J.N., Zhu, J., Yu, Y., Teng, X.D., Lou, Y., Xu, X.F., Liu, J.L., Yang, Z.N. 2014. Dyt1 directly regulates the expression of TDF1 for tapetum development and pollen wall formation in Arabidopsis. Plant J. 80:10051013.

Hu, J., Huang, W.C., Huang, Q., Qin, X.J., Yu, C.C., Wang, L.L., Li, S.Q., Zhu, R.S., Zhu, Y.G. 2014. Mitochondria and cytoplasmic male sterility in plants. Mitochondrion. 19:282-288.

Hu, Y.M., Tang, J.H., Yang, H., Xie, H.L., Lu, X.M., Niu, J.H., Chen, W.C. 2006. Identification and mapping of $R f-I$ an inhibitor of the $R f 5$ restorer gene for Cms-C in maize (Zea mays L.). Theor. Appl. Genet. 113:357-360.

Kamps, T.L., Chase, C.D. 1997. RFLP mapping of the maize gametophytic restorer-of-fertility locus ( $r f 3)$ and aberrant pollen transmission of the nonrestoring rf3 allele. Theor. Appl. Genet. 95:525-531.

Kaul, M.L.H. 1988. Male Sterility in Higher Plants. Springer. Berlin Heidelberg.

Lee, S.L.J., Gracen, V.E., Earle, E.D. 1979. The cytology of pollen abortion in C cytoplasmic male-sterile corn anther. Am. J. of Bot. 66:656-667.

Levings, C.S. 1990. The Texas cytoplasm of maize: cytoplasmic male sterility and disease susceptibility. Science, 250:942-947.

Li, L., Li, Y.X., Song, S.F., Deng, H.F., Li, N., Fu, X.Q., Chen, G.H., Yuan, L.P. 2015. An anther development f-box (ADF) protein regulated by tapetum degeneration retardation (TDR) controls rice anther development. Planta. 241:157-166.

Liu, Y.M., Zhao Z.F., Lu Y.L., Li. C., Wang, J., Dong, B.X., Liang, B., Qiu, T., Zeng, W.B., Cao, M.J. 2016. A preliminary identification of Rf*-A619, a novel restorer gene for CMS-C in maize (Zea mays L.). PeerJ. 4:e2719; DOI 10.7717/peerj.2719.

Liu, Z.Y., Peter, S.O., Long, M.H., Weingartner, U., Stamp, P., Kaeser, O. 2002. A PCR assay for rapid discrimination of cytoplasm types in maize. Crop Science. 42:566-569.

Luan, J., Liu, T.R., Luo, W.Q., Liu, W., Peng, M.Q., Li, W.J., Dai, X.J., Liang, M.Z., Chen, L.B. 2013. Mitochondrial DNA genetic polymorphism in thirteen rice cytoplasmic male sterile lines. Plant Cell Rep. 32:545-554.

Ma, H. 2005. Molecular genetic analyses of microsporogenesis and microgametogenesis in flowering plants. Plant Biol. 56:393-434.

Moon, J., Skibbe, D., Timofejeva, L., Wang, C.J., Kelliher, T., Kremling, K., Walbot, V., Cande, W.Z. 2013. Regulation of cell divisions and differentiation by male sterility32 is required for anther development in maize. Plant J. for Cell and Mol. Biol. 76:592-602.

Nan, G.L., Zhai, J., Arikit, S., Morrow, D., Fernandes, J., Mai, L., Nguyen, N., Meyers, B.C., Walbot, V. 2017. MS23, a master basic helix-loop-helix factor, regulates the specification and development of the tapetum in maize. Development. 144:163-172.

Qin, T.C., Xu, M.L., Dun, D.X. 1990. Cytoplasmic male-sterility: identification of the number of the restorer genes. Maize Genet. Coop. News Let. 64:124.

Ren, R.H., Nagel, B.A., Kumpatla, S.P., Zheng, P.Z., Cutter, G.L., Greene, T.W., Thompson, S.A. 2012. Maize cytoplasmic male sterility (cms) c-type restorer RF4 gene, molecular markers and their use. US Patent 20120090047, April 12. United States Patent and Trademark Office, United States. Available at https:// www.google.com/patents/ US20120090047.

Rhoades, M.M. 1933. Cytoplasmic inheritance of male sterility in Zea mays. J. of Genet. 73:71-93.

Sisco, P.H. 1991. Duplications complicate genetic mapping of $R f 4$, a restorer gene for CMS-C cytoplasmic male sterility in corn. Crop Sci. 31:1263-1266.

Skibbe, D.S., Schnable, P.S. 2005. Male sterility in maize. Maydica. 50:367-376.

Slischuk, G.I., Kozhukhova, N.E., Sivolap, Y.M. 2011. Molecular genetic analysis of maize mitochondrial regions associated with CMS. Cytol. and Genet. 45:143-147.

Tang, J.H., Fu, Z.Y., Hu, Y.M., Li, J.S., Sun, L.L., Ji, H.Q. 2006. Genetic analyses and mapping of a new thermo-sensitive genic male sterile gene in maize. Theor. Appl. Genet. 113:11-15.

Warmke, H.E., Lee, S.J. 1978. Pollen abortion in T cytoplasmic male-sterile corn (Zea mays): a suggested mechanism. Science. 200:561-563.

Wilson, Z.A., Zhang, D.B. 2009. From Arabidopsis to rice: pathways in pollen development. J. Exp. Bot. 60:1479-1492. 
Wise, R.P., Bronson, C.R., Schnable, P.S., Horner, H.T. 1999. The genetics, pathology, and molecular biology of T-cytoplasm male sterility in maize. Adv. in Agron. 65:79-83.

Wu, Y.Z., Fox, T.W., Trimnell, M.R., Wang, L.J., Xu, R.J., Cigan, A.M., Huffman, G.A., Garnaat, C.W., Hershey, H., Albertsen, M.C. 2015. Development of a novel recessive genetic male sterility system for hybrid seed production in maize and other cross-pollinating crops. Plant Biotechnol. J. 14:1046-1054.

Zabala, G., Gabaylaughnan, S., Laughnan, J.R. 1997. The nuclear gene Rf3 affects the expression of the mitochondrial chimeric sequence R implicated in S-type male sterility in maize. Genet. 147:847-60.

Zhao, H.X., Li, Z.J., Hu, S.W., Sun, G.L., Chang, J.J., Zhang, Z.H. 2010. Identification of cytoplasm types in rapeseed (Brassica napus L.) accessions by a multiplex PCR assay. Theoretical and Applied Genetics. 121:643-650.

Zheng, Y.L. 1982. Study on the mechanism of the fertility about several types of cytoplasmic male sterility in maize (Zea mays, L.). Journal of Huazhong Agricultural College. 1:44-68. (in Chinese with English abstract).

Zhou, H., Liu, Q.J., Li, J., Jiang, D.G., Zhou, L.Y., Wu, P., Lu, S., Li, F., Zhu, L.Y., Liu, Z.L., Chen, L.T., Liu, Y.G., Zhang, C.X. 2012. Photoperiod-and thermo-sensitive genic male sterility in rice are caused by a point mutation in a novel noncoding RNA that produces a small RNA. Cell Research. 22:649-660.

\section{Electronic Supplementary Material (ESM)}

Electronic Supplementary Material (ESM) associated with this article can be found at the website of CRC at http://www.akademiai.com/content/120427/

\section{Electronic Supplementary Table S1. Testers used in this study}

Electronic Supplementary Table S2. Fertility expression of K932MS in different locations and years

Electronic Supplementary Table S3. Classification of fertility expression of hybrid progenies from different male parents

Electronic Supplementary Table S4. Fertility expression of $\mathrm{BC}_{1}$ generation

Electronic Supplementary Table S5. Fertility expression of $\mathrm{BC}_{2}$ generation

Electronic Supplementary Figure S1. Comparisons of K932F with K932S: (a) tassel; (b) spikelet; (c) anthers; (d) pollens stained with $\mathrm{I}_{2}-\mathrm{KI}$, fertile pollens were stained dark, but the sterile one unstained. F: fertile; S: sterile

Electronic Supplementary Figure S2. Discrimination of sterile cytoplasm type by PCR using cytoplasmic typespecifically primers. M: marker; 1 : maintainer line Mo17; 2: $\mathrm{CMS}_{-\mathrm{C}_{\mathrm{Mo17}}} ; 3: \mathrm{CMS}_{\mathrm{Mo17}} ; 4: \mathrm{CMS}_{\mathrm{Mo17}} ; 5$ and 6: K932S; 7 and 8: K932F. cms-C amplified $\mathrm{C}$ type band, cms-S amplified $\mathrm{S}$ type band, cms-T amplified T type band

Electronic Supplementary Figure S3. K932MS anther transection. Fertility anther and meiocyte: a, b, c, d. Sterility anther and meiocyte: e, f, g, h. a, e: the early period of the life of a pollen mother cell $(\times 100)$; b, f: the later period of the life of a pollen mother cell $(\times 100)$; c, g: the dyad period $(\times 200)$; $d$, h: the microspore period $(\times 200)$. Arrows highlight the tapetum. The scale bar represents $10 \mu \mathrm{m}$

Electronic Supplementary Figure S4. Meiocyte behaviour of K932MS (×400). Fertility meiocyte: a, b, c, d. Sterility meiocyte: e, f, g, h. The scale bar represents $2 \mu \mathrm{m}$ 